Nonlin. Processes Geophys., 21, 1-8, 2014

www.nonlin-processes-geophys.net/21/1/2014/

doi:10.5194/npg-21-1-2014

(c) Author(s) 2014. CC Attribution 3.0 License.

(C) (i)

\title{
Power law statistics of force and acoustic emission from a slowly penetrated granular bed
}

\author{
K. Matsuyama ${ }^{1}$ and H. Katsuragi ${ }^{2}$ \\ ${ }^{1}$ Department of Applied Science for Electronics and Materials, Kyushu University, 6-1 Kasuga 816-8580, Japan \\ ${ }^{2}$ Department of Earth and Environmental Sciences, Nagoya University, Furocho, Chikusa, Nagoya 464-8601, Japan
}

Correspondence to: H. Katsuragi (katsurag@eps.nagoya-u.ac.jp)

Received: 18 April 2013 - Revised: 29 July 2013 - Accepted: 6 November 2013 - Published: 3 January 2014

\begin{abstract}
Penetration-resistant force and acoustic emission (AE) from a plunged granular bed are experimentally investigated through their power law distribution forms. An AE sensor is buried in a glass bead bed. Then, the bed is slowly penetrated by a solid sphere. During the penetration, the resistant force exerted on the sphere and the AE signal are measured. The resistant force shows power law relation to the penetration depth. The power law exponent is independent of the penetration speed, while it seems to depend on the container's size. For the AE signal, we find that the size distribution of $\mathrm{AE}$ events obeys power laws. The power law exponent depends on grain size. Using the energy scaling, the experimentally observed power law exponents are discussed and compared to the Gutenberg-Richter (GR) law.
\end{abstract}

\section{Introduction}

Power law nature is one of the most ubiquitous and fundamental features in various natural phenomena (Turcotte, 1997). For seismic activity, the well-known GutenbergRichter (GR) law (Gutenberg and Richter, 1949) is a typical power law relation. In spite of the global applicability of the GR law, its origin has not been clarified yet. A lot of efforts have been devoted to exploring the governing mechanics of the GR law. Examples include fractal characterization (Turcotte, 1997), unified scaling analysis (Bak et al., 2002), numerical simulation (Olami et al., 1992), etc. Recently, experiments and simulations of soft materials such as polymer gel and granular matter have been performed to mimic seismic activity. Yamaguchi et al. have studied the sliding friction of a polymer gel slab and found a power law distribution of the force drop (Yamaguchi et al., 2011). By a numerical simulation of slowly sheared granular matter, avalanche magnitude frequency has been examined and the GR-like power law relation has been observed (Hatano et al., 2011). While these situations are quite different from actual seismicity, the results might be informative to explain something essential for universal power law distribution.

Particularly, granular matter can be regarded as a kind of heterogeneous media consisting of a lot of discrete units. Recently, the fundamental frictional properties of granular matter have been studied intensively to understand the complex nature of earthquakes (Kuwano and Hatano, 2011; Hatano and Kuwano, 2013). The shear banding and granular bearing effect in the fault zone have also been studied ( $₫$ ström et al., 2000, 2001). Wave propagation and scattering in heterogeneous media is a key factor in analyzing the seismic data (Sato and Fehler, 1997). The wave propagation within confined granular matter has been studied experimentally (Jia et al., 1999; Jia, 2004). In the studies, P-, S-, and scattered waves have been observed in the highly confined granular matter. The exponential decay of the intensity of scattered waves has also been reported by Jia (2004). In general, granular matter is very useful for investigating the statistical properties of a certain class of macroscopically heterogeneous material. Typical size of geometrical heterogeneity in static granular structure roughly corresponds to grain size. We use granular matter as a prototype material of geometrically heterogeneous materials.

To characterize the granular behavior, acoustic emission (AE) measurement is used in this study. Weak elastic waves due to the micro-crack opening or micro-slipping in a continuum specimen can be detected by an $\mathrm{AE}$ sensor. The $\mathrm{AE}$ method is a powerful nondestructive testing tool (Grosse and Ohtsu, 2008) that has been applied to the characterization 
of geomaterial fracture, too. The AE activity clearly relates to the fracturing process of rocks (Mogi, 1962; Zang et al., 1996; Mogi, 2007). The AE measurement has also been applied to the granular matter. Hidalgo et al. have measured the AE signal from a uniaxially-compressed granular column (Hidalgo et al., 2002). In the previous granular studies (Hidalgo et al., 2002; Jia et al., 1999; Jia, 2004), granular specimens have been highly confined. Contrastively, we would like to focus on the loose situation in this study. Acoustic measurement in two-dimensional loose granular packing has been carried out with photoelastic disks (Owens and Daniels, 2011, 2013). Sound propagation and force chain structure were related by nonlinear scaling. While the photoelastic measurement has a great advantage in seeing the actual force chain network directly, it is limited in twodimensional analysis. Moreover, AE event statistics in loose granular matter have not been measured yet. In this study, we measure and analyze the AE event statistics in threedimensional loose granular matter. Furthermore, loose granular matter shows various interesting phenomena such as convection, size segregation, avalanche, and so on (Duran, 2000; Mehta, 2007). The bulk characteristics of granular matter themselves are interesting enough. In addition, local rearrangements and/or slips of grains, which can be the source of AE events, occur very frequently in the penetrated granular matter.

The AE measurement corresponds to microscopic characterization of the loaded granular bed. On the other hand, the macroscopic resistant force against the penetration is one of the most fundamental quantities to characterize the penetrated granular bed. Resistant force measurement has been carried out in some previous investigations (Stone et al., 2004; Hill et al., 2005; Katsuragi, 2012a, b). These studies have revealed the complex relation between penetration depth and resistant force. To obtain the macroscopic relation, they have regarded the bulk granular matter as a continuum. Macroscopic resistant force is also measured and discussed in this experiment. The origin of resistant force must be frictional supports at the grain contact points. By breaking such supports, the penetration proceeds. This is more or less similar to the origin of an earthquake that is the slip of a fault. In this study, we would like to examine the microscopic fracture of loose granular structure using the AE method. The statistical property of the AE events is particularly focused. Power law nature of the AE statistics is found and discussed by the mode of deformation/fracturing.

\section{Experimental setup}

We use a simple experimental apparatus as shown in Fig. 1. A cylindrical plexiglass container of inner radius $R=150 \mathrm{~mm}$ is filled with roughly monodisperse and spherical glass beads whose diameter $d$ is $0.4,0.8$, or $2 \mathrm{~mm}$ (AS-One Corp. BZ-04, BZ-08, and BZ-2). Height of the granular bed $H$ is always

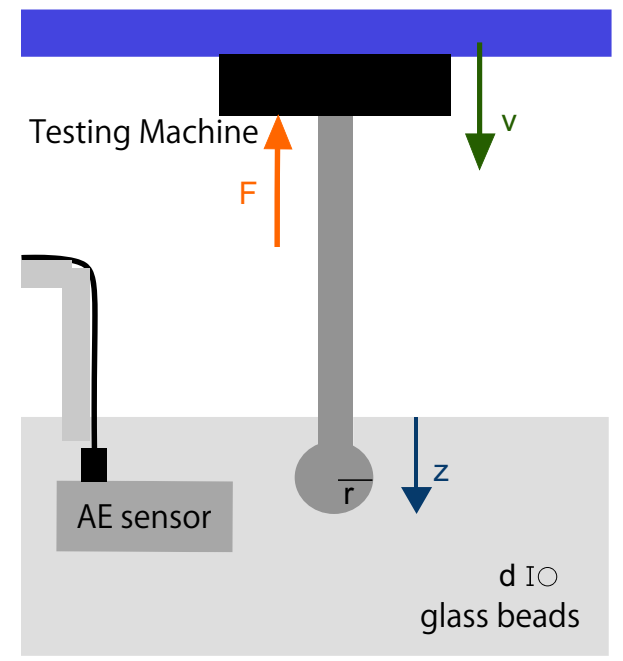

Fig. 1. Schematic illustration of the experimental setup. An AE sensor is buried in a glass bead bed (grain size $d$ ). A sphere (radius $r$ ) slowly penetrates into the bed. The exerted force $F$ and AE amplitude $A$ are measured.

set as $H=100 \mathrm{~mm}$. The bed is prepared by sedimentation through a sieve of $4 \mathrm{~mm}$ opening. During the filling, an AE sensor (NF AE-900s-WB) is buried and fixed in the bed. The position of the sensor's head is about $20 \mathrm{~mm}$ deep from the surface and $\delta=30 \mathrm{~mm}$ away from the center of the container. The sensor is connected to the amplifier and the acquisition system consisting of NF AE-9913, NF AE9922, NI PCIe6251 , and a PC. The top surface of the granular bed is open to the atmosphere. Note that any confining pressure is not applied to the bed. Thus, the granular bed is very loose and deformable. In this study, we use only one AE sensor. To locate the source of AE events, plural (at least 3) sensors are necessary. However, it might be difficult to detect the AE events occurring far from the sensor, since the loose granular bed is very dissipative. As a first step of the AE statistics measurement for the penetrated granular bed, we bury an AE sensor in the bed.

After the bed preparation, a steel sphere of radius $r=5$, 10 , or $20 \mathrm{~mm}$ is slowly plunged into the bed. The penetration speed is fixed as $v=0.5,1.0$, or $5.0 \mathrm{~mm} \mathrm{~s}^{-1}$, using a universal testing machine (Shimadzu AG-100NX). The resistant force $F$ exerted on the sphere and the AE amplitude $A$ are measured. Sampling rates of $F$ and $A$ are $5 \mathrm{kS} \mathrm{s}^{-1}$ and $1 \mathrm{MS} \mathrm{s}^{-1}$, respectively. The penetration depth $z$ is defined as the distance between the bottom of the penetrating sphere and the initial surface level of the granular bed, i.e., $z=0$ corresponds to the point at which the bottom of the sphere touches the surface of the granular bed. This experimental setup is similar to the previous works (Katsuragi, 2012a, b). The only difference is the container's radius. That is much greater than the previous one. 


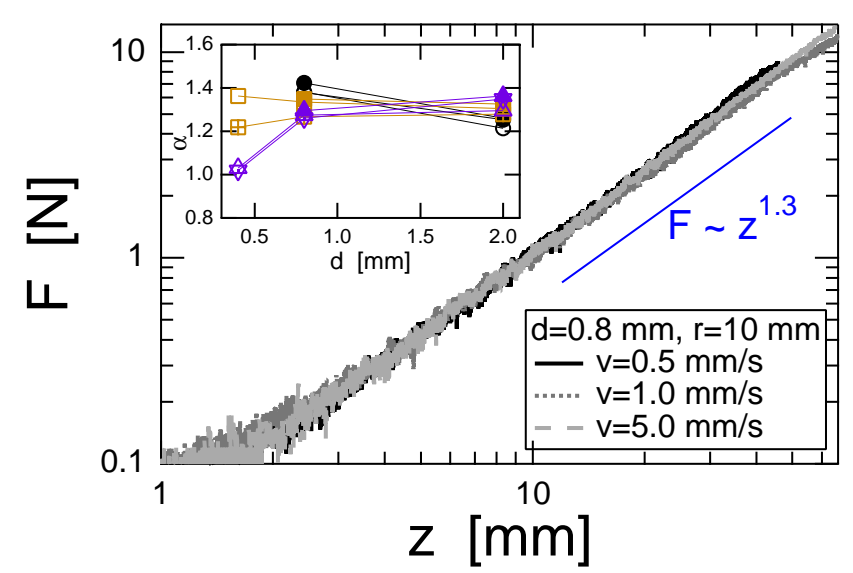

Fig. 2. Resistant force $F$ exerted on the spherical intruder as a function of penetration depth $z$. Different penetration speed data collapse to a scaling relation, $F \sim z^{1.3}$. The inset shows power law exponents for various experimental conditions. Color and mark codes for the inset plot are the same as those in Fig. 4b.

To collect a sufficient number of AE events, we have to bury the $\mathrm{AE}$ sensor in the granular bed. If we just put the sensor on the top surface of the bed, only a very weak signal is detected. This means that the whole sensor's body works as a wave receiver. Besides, dissipation of elastic waves in loose granular matter is significant. Therefore, the sensor is able to capture AE events occurring only on the boundary or in the vicinity of it. We can regard the AE sensor as a huge artificial fault (slip face) embedded in the granular bed, and the grain rearrangements and/or slips on the boundary or in the vicinity of the fault are measurable by the sensor. Using this experimental setup, we would like to study the loose granular deformation characteristics around the artificial slip face.

\section{Results}

First, we are going to focus on the resistant force. Example data of $F(z)$ with three different penetration speeds are displayed in Fig. 2. It is confirmed that all the data collapse to a simple power law relation, $F \sim z^{\alpha}$. Similar resistant force scaling has been reported by some research groups (Stone et al., 2004; Hill et al., 2005; Katsuragi, 2012a, b). However, the value of $\alpha$ ranges from 1 to 1.5 depending on the experimental conditions. For the data shown in Fig. 2, $\alpha$ is about 1.3. While the experimental setup of Katsuragi (2012a, b) was very similar to the current study, the previous study resulted in $\alpha \simeq 1.5$. The main difference among these experiments is the container's radius. Thus we consider that the strength of the nonlinearity (value of $\alpha$ ) depends on the container's size effect. In the previous study (Katsuragi, 2012a, b), the container's radius $R$ has been fixed, and the obtained scaling law has been free from $R$. We consider that the container's size effect corresponds to the side wall effect. More
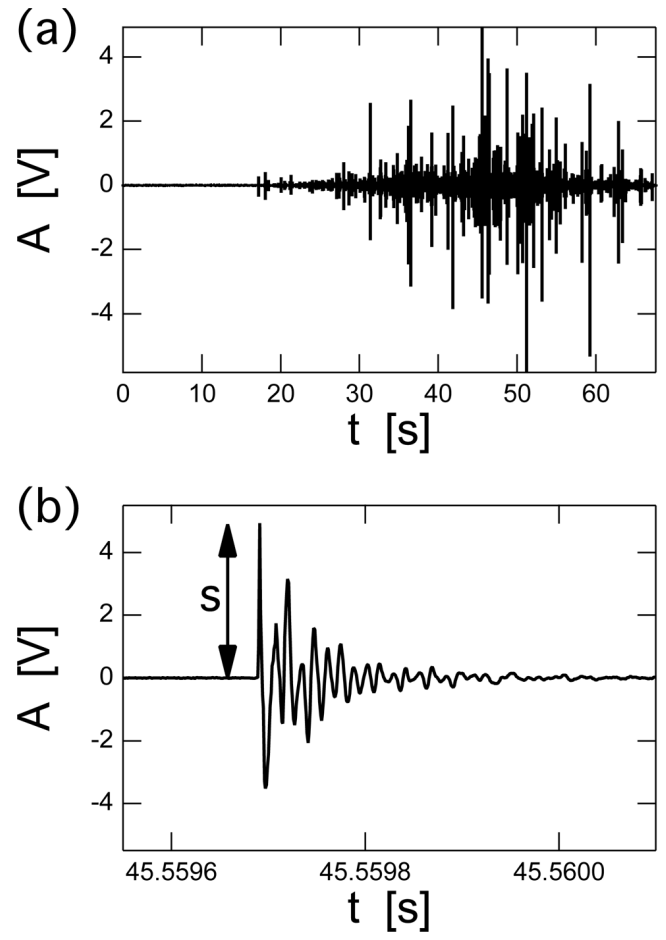

Fig. 3. Raw data example of AE signal. Experimental conditions are $d=0.8 \mathrm{~mm}, r=10 \mathrm{~mm}$, and $v=1.0 \mathrm{~mm} \mathrm{~s}^{-1} . t=0$ corresponds to $z=0$. Whole data during a penetration is shown in (a), and an example of a single AE event is shown in (b). Size of AE event $s$ is defined by the maximum amplitude.

systematic experiments with varying $R$ are necessary to obtain the quantitative size dependence of the scaling exponent. In the limit of $R \rightarrow \infty, \alpha$ should be unity because the wall effect could be negligible.

The $\alpha$ values computed by the least square fitting for various experimental conditions are shown in the inset of Fig. 2. Almost all the data show $\alpha \simeq 1.3$, since $R$ is fixed as $R=150 \mathrm{~mm}$. The only data point showing $\alpha \simeq 1$ corresponds to the case of $d=0.4 \mathrm{~mm}$ (the smallest beads) and $r=20 \mathrm{~mm}$ (the largest sphere). In such a case, the deformation might be localized around the proximity of the penetrating sphere. Then, the side wall effect becomes negligible and penetration resistance can be roughly expressed by hydrostatic-like depth-proportional force. However, note that the order of resistant force is approximately one order of magnitude greater than the hydrostatic expectation. Such a large depth-dependent resistant force has already been observed in the granular impact experiment (Katsuragi and Durian, 2007, 2013). Since the narrower container shows the stronger nonlinearity (larger $\alpha$ value), the origin of nonlinear slow penetration resistance is different from the Janssen effect (Janssen, 1895; Sperl, 2006).

Next, the AE amplitude data are analyzed. A raw data example of the AE signal $A(t)$ is shown in Fig. 3. Figure 3 a displays a whole time series of a penetration. $t=0$ 

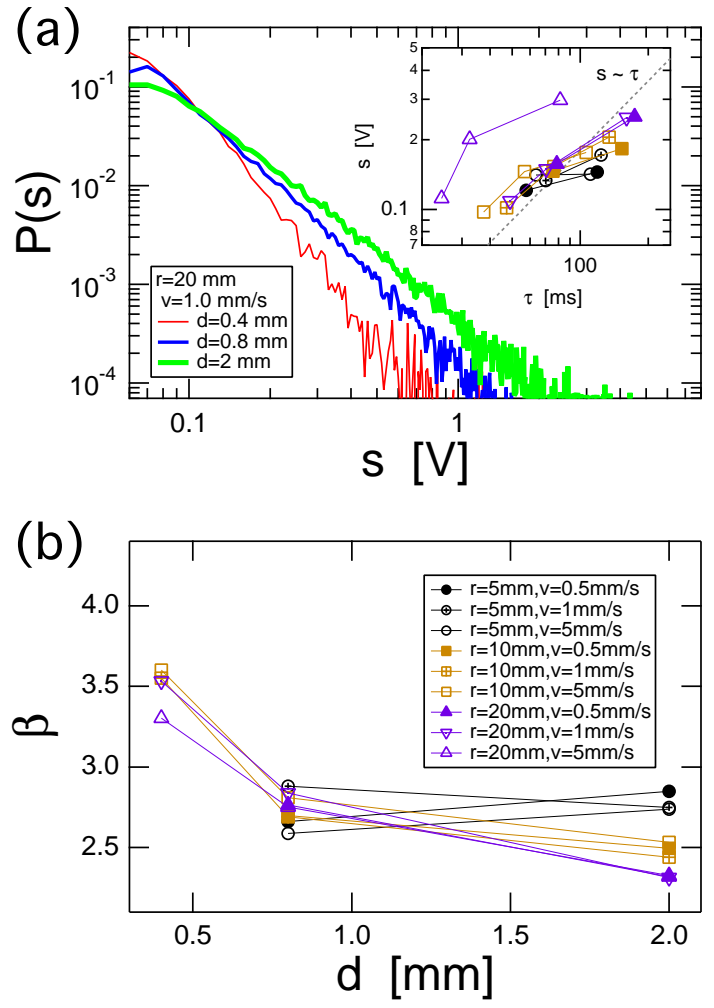

Fig. 4. Size distribution of AE events. (a) Normalized AE frequency $P(s)$ shows power law form, $P(s) \sim s^{-\beta}$. The inset shows the relation $s \sim \tau$, where $\tau$ is the decay timescale of AE events. (b) Experimental conditions dependence of the exponent $\beta$. The exponent $\beta$ depends on grain size $d$.

corresponds to the time of $z=0$. Experimental conditions are $d=0.8 \mathrm{~mm}, r=10 \mathrm{~mm}$, and $v=1.0 \mathrm{~mm} \mathrm{~s}^{-1}$. A large number of bursts can be identified in the AE signal. In the early regime $(t<20 \mathrm{~s}$, i.e., $z<20 \mathrm{~mm})$, only very weak signals are detected. Most of all, significantly large AE events are found in the deep penetration regime. The same tendency can be qualitatively confirmed for all other experimental conditions. This might mean that the granular bed has to be compressed down to a certain level of compaction in order to emit the detectable AE signal. The AE signals from extremely fluffy granular beds cannot be detected even by recent $\mathrm{AE}$ techniques. While we consider that the measured $\mathrm{AE}$ events come from the vicinity of the sensor, a little compression by the penetration is needed to detect the events. Note that, however, the granular bed is still easily deformed at the sensor's place because no additional loading is applied to the free surface of the granular bed.

In this paper, we are going to focus on the statistical property of the AE events. Thus, the measured $\mathrm{AE}$ amplitude data must be divided into each $\mathrm{AE}$ event. We use a threshold value $V_{\text {th }}=0.06 \mathrm{~V}$ and a dead time $t_{\text {dead }}=0.3 \mathrm{~ms}$ to separate $\mathrm{AE}$ events. These specific values are determined by the data quality such as noise level and signal decay timescale. In Fig. 3b, an AE event picked up from Fig. 3a is shown. The AE event shows typical attenuating oscillation. The total number of $\mathrm{AE}$ events identified by this procedure ranges from $10^{3}$ to $10^{4}$. To characterize the AE events, the size of each event $s$ is defined by the maximum amplitude as shown in Fig. 3b. The size distribution of AE events is the main interest of this study.

The obtained size distribution of $s$ is shown in Fig. 4a. The size distribution clearly obeys power law form $P(s) \sim s^{-\beta}$, where $P(s)$ and $\beta$ are a normalized number (relative frequency) of $\mathrm{AE}$ events of size $s$ and a characteristic power law exponent, respectively. In Fig. $4 \mathrm{~b}$, the values of $\beta$ computed by the least square fitting for various experimental conditions are plotted. As can be seen in Fig. $4 \mathrm{~b}, \beta$ is almost independent of penetration speed $v$ and penetrator radius $r$. Instead, $\beta$ depends on grain size $d$. This $d$-dependent $\beta$ can be confirmed in Fig. $4 \mathrm{a}$ as well. For $d=0.8$ and $2 \mathrm{~mm}, \beta$ distributes around 2.5-3.0, while it is about 3.5 for $d=0.4 \mathrm{~mm}$. This means that the relative frequency of larger AE events decreases for the smaller grains bed. Note that the glass beads used in this study are almost monodisperse. They do not possess power law size distribution. Nevertheless, the size distribution of microscopic rearrangements and/or slips show the clear power law form. Since the granular matter consists of a lot of discrete particles, the internal force structure (force chain structure) shows very inhomogeneous distribution. The nature of power law statistics could relate to this inhomogeneity of the force chain structure.

\section{Discussion}

Power law size distribution of AE events is universal (Mogi, 2007; Grosse and Ohtsu, 2008). The power law exponent is a key quantity in characterizing the state of the specimen. In this study, we observe the power law distribution of $\mathrm{AE}$ events from a plunged granular bed. Here we assume that an $\mathrm{AE}$ event comes from a microscopic fracturing or slipping occurring in the specimen. In order to discuss the similarity between the granular AE measurement and other fracturinglike earthquakes, the power law exponent should be compared. We discuss the similarities and differences between granular $\mathrm{AE}$ and earthquakes or other $\mathrm{AE}$ in what follows this section. First, simple comparison of power law exponents is discussed by the energy scaling. Then, serious differences between granular $\mathrm{AE}$ and actual earthquakes are qualitatively discussed, based on the recent electromagnetic emission measurement.

The GR law is written as $\log n(M)=a-b M$, where $n(M)$ is the frequency of earthquakes of magnitude $M$, and $a$ and $b$ are constants. Particularly, $b$ is important since it relates to the power law exponent $\beta$. Moreover, most of the $b$ values for real seismic activity along the active fault zones are roughly 1 . Traditionally, the $b$ value has been related to $\beta$ as $b=\beta-1$ 
by the rock AE measurements (Mogi, 1962; Scholz, 1968). Here we reconsider the relation of the exponents in terms of the corresponding energy scaling. Let us consider the number of earthquakes $n(M) d M$ in the range of magnitude ( $M$, $M+d M)$. Using the relation $\log E_{S}=C_{E}+(3 / 2) M$ (Scholz, 2002), $n(M) d M$ can be transformed to $n\left(E_{s}\right) d E_{s}$ as

$n(M) d M \sim E_{S}^{-2 b / 3} d\left(\log E_{S}\right) \sim E_{S}^{-\left(\frac{2}{3} b+1\right)} d E_{S}$,

where $E_{S}$ and $C_{E}$ are the seismic energy and a constant.

Next, we estimate the energy of $\mathrm{AE}$ events, $E_{A}$. In principle, $E_{A}$ is proportional to the integral over signal amplitude. In order to estimate $E_{A}$ solely by $s$, we examine the relation between $s$ and the decay timescale of the envelope of $\mathrm{AE}$ events. In the inset of Fig. 4a, the relations between $s$ and the AE event's decay timescale $\tau$ for various experimental conditions are presented. $\tau$ is computed from the fitting of the $\mathrm{AE}$ event's envelope to $A \sim \exp \left[-\left(t-t_{0}\right) / \tau\right]$, where $t_{0}$ is the beginning time of the event. As seen in the inset of Fig. 4a, the proportional relation $s \sim \tau$ is almost satisfied. This relation enables us to estimate $E_{A}$ by a simple scaling, $E_{A} \sim s^{2}$. Using this maximum amplitude-squared proportional energy $E_{A}$, the number of $\mathrm{AE}$ events in the range of $(s, s+\mathrm{d} s)$ can be written as

$P(s) \mathrm{d} s \sim E_{A}^{-\frac{\beta+1}{2}} d E_{A}$.

Assuming $E_{S} \sim E_{A}$, we finally obtain

$b=\frac{3}{4}(\beta-1)$.

This relation is slightly different from the traditional one, $b=\beta-1$. It should be noticed that Eq. (3) is obtained mainly by the assumption of $E_{A} \sim s^{2}$. Substituting representative values $\beta=2.5$ and 3.5 into Eq. (3), corresponding $b$ values are calculated as 1.1 and 1.9. The smaller value $b=1.1$ is roughly consistent with the common $b$ value of the real seismic data.

In the smaller grains case $(d=0.4 \mathrm{~mm})$, the corresponding $b$ value $(\simeq 1.9)$ is greater than the usual GR law. In general, the power law exponent of $\mathrm{AE}$ event distribution depends on the mode of fracture. The value of $\beta$ for plastic deformation is greater than that for brittle fracture. For example, Imaeda et al. have reported that the AE power law exponent depends on metal crystal structure, which relates to the degree of plasticity (Imaeda et al., 1976). Recent studies have also found power law AE statistics and focused on dislocation processes in the materials (Richeton et al., 2005; Dimiduk et al., 2006). Moreover, magnetic noise from a dynamic fracture shows a similar trend (Kun et al., 2004). The larger power law exponent has been observed in ductile fracture rather than in brittle fracture. Applying this idea to the current experimental result, the deformation of smaller grains is closer to plastic rather than brittle.
The grain diameter $d$ can be regarded as a typical size of the static heterogeneity. As mentioned before, we consider that the sensor corresponds to a slip face in the current experimental setup. Neglecting the detailed shape effect of the sensor, the size ratio between the sensor and grains can be utilized to characterize the state. The longer dimension of the sensor we use is $L=40 \mathrm{~mm}$. Then, we can consider the ratio $d / L=0.02(=0.8 \mathrm{~mm} / 40 \mathrm{~mm})$ to be a marginal point between brittle-like and plastic-like behaviors.

For compressed rock samples, the $b$ value depends on the phase of fracture (Zang et al., 1996). A significant drop in $b$ values has been observed just before the main fracture. The drop in $b$ values means that the fracture mode becomes more brittle, and the relative frequency of large rare events increases. This might come from the growth of the size of homogeneities in the specimen, i.e., the effective grain size increases as it approaches the main fracture. Then, the compressed rock result is qualitatively consistent with the current study. In Mogi (1962), the $b$ value increases as the heterogeneity size in the specimen grows. Mogi (1962) used the volume fraction of granular pumice in the rock specimen to characterize the size of heterogeneity. That result can be understood if we assume that the porous pumice makes the specimen plastic.

The power law exponent of size distribution depends on the degree of dissipation (Olami et al., 1992; Ramos, 2011). Small grains are more dissipative than large grains. The emitted AE signal could be dissipated at the grain contacts. The amount of grain contact in small-grains beds is greater than that in large-grains beds. Then, the large AE events cannot grow in a small-grains bed due to the significant dissipation. In other words, large $\mathrm{AE}$ events might be screened by the dissipation. The large AE events correspond to large slips of grains and cause discontinuous (brittle-like) slips. Since such large events are suppressed in small-grains beds by the significant dissipation, global deformation of small-grains beds becomes rather continuous. Put another way, the dissipative small-grains deformation consists of smaller avalanches and results in a large $\beta$ value. In the dissipative small-grains system, therefore, continuous (plastic) deformation is attained. This is contrastive to the large-grains bed in which the larger avalanches of slipping result in large-scale discrete (brittle) fracturing. Thus the understandings of the power law exponent by dissipation and by the mode of fracturing might be two sides of the same coin. The one side is the fracture of continuum, and the other side is the granular deformation or slips.

In the experiment, the measured power law exponent depends on $d$ rather than the ratio of $r / d$. From the viewpoint of similitude, we should fix the ratios $R / d$ and $\delta / d$ as well as $r / d$, in order to evaluate the similarity of various grain size systems. We already checked that $\beta$ is almost independent of $r$ (Fig. 4b). In addition, we also checked that the power law exponent is independent of $\delta / d$ in some $\delta$ cases. However, the container's radius $R$ is fixed in this experiment, i.e., $R / d$ 
is not fixed. $R$ (and the corresponding curvature) may play an important role. In quasi-static sheared granular matter, the frictional property depends on the ratio radii of grains and cells (Kamrin and Koval, 2012). As we discussed before, the penetration-resistant force is affected by the container's side wall. The AE statistics might also be affected by it. To clarify the actual mechanism determining the varying exponent $\beta$, the systematic comparisons of the current result with the simply sheared or highly compressed systems are necessary. Such detailed characterization is open to future study.

In the current study, only the AE events are measured. To characterize the mode of fracturing more precisely, electromagnetic emission (EME) should also be measured simultaneously. According to the previous works (Eftaxias and Potirakis, 2013; Mori et al., 2004, 2009; Baddari and Frolov, 2011), there are two kinds of AE signals: (i) AE signals with simultaneous EME that correspond to the creation of new surfaces, and (ii) AE events without EME corresponding to frictional noise of existing surfaces. While the former is closer to real earthquake events, the current experimental condition probably corresponds to the latter. Thus, the mechanics level agreement between deformed granular matter and real seismic activity is very limited. Moreover, the power law exponents obtained by other geological AE measurements are very different from the current result (e.g., Kapiris et al. (2004)). These features might be critical differences between the current granular $\mathrm{AE}$ and the other previous $\mathrm{AE}$ measurements. The penetrated granular bed probably belongs to a different class from usual solids, due to its inherent discreteness. While the similarity is limited, here we have argued for the power law exponents along the lines of energy scaling of the GR law. On the other hand, to mimic the geological scale phenomena by tabletop experiment, the model soil strength should be very small. Therefore, the sand box can be a good model for the geological scale phenomena (e.g., Yamada et al. (2010)). By means of similarity modeling and dimensional analysis, the current experimental result may be relevant to geological scale phenomena. The detailed statistical comparison is necessary for validating the relation between the loose granular experiment and the actual seismic activity. The current experimental result cannot conclude the complete similarity at this time.

So far, we have discussed $F$ and $A$ independently. In order to discuss their direct relation, we compute the crosscorrelation between $\Delta F(t)$ and $A(t)$, where $\Delta F(t)$ is defined by $F(t)-k t^{\alpha}$ ( $k$ is a fitting parameter). However, it is hard to observe a clear correlation between them. While the source of $\Delta F(t)$ is grain rearrangement and/or slip at the penetrator boundary, the source of the detected AE event is localized in the vicinity of the AE sensor. That is why the clear correlation cannot be confirmed.

The waiting time $\Delta t$ between two successive AE events might also bring useful information. However, the measured $\Delta t$ distribution obeys neither power law nor exponential form. The detailed characterization of $\Delta t$ is the most important future problem.

In this experiment, we regard the AE sensor itself as a slip face embedded in the granular specimen, and we measured the AE signal during the penetration. This is quite different from canonical usage of the AE technique. Fundamental characterizations are necessary to fully reveal the underlying physics of granular AE phenomena. For example, the measurement of single grain slip on the sensor's surface and other geometry like simple shears should be investigated to obtain quantitative conclusions for granular AE measurement. In this study, we have mainly focused on the power law properties of $F$ and $A$.

\section{Conclusions}

The resistant force $F$ and AE amplitude $A$ in a plunged granular bed was investigated. The force $F$ exhibits a power law relation to the penetration depth $z$ as $F \sim z^{\alpha}$. The value of $\alpha$ obtained in this experiment is about 1.3. This value ranges from 1 to 1.5 , depending on the experimental conditions. Particularly, the container's size is the most important parameter, since $\alpha$ seems to be mainly determined by the side wall effect. For the AE signal, we found that the size distribution of the maximum amplitude $s$ obeys the power law whose exponent $\beta$ ranges from 2.5 to 3.5 . By the dimensional analysis, this exponent was compared with the $b$ value of the GR law. The length ratio between heterogeneity (grain size) and the embedded fault (sensor) $d / L$ is a possible parameter to characterize the system. The large grain $(d / L \geq 0.02)$ bed shows $b \simeq 1$, while the smaller grains $(d / L<0.02)$ bed shows $b \simeq 2$. To evaluate the details of micro-earthquake-like behaviors occurring in the loose granular matter, experiments with other conditions and simultaneous EME measurement are required. The current experiment is just a first-step approach to the micro-seismicity of easily deformed loose granular matter.

Acknowledgements. The authors greatly appreciate T. Hatano, F. Kun, A. Onuki, H. Kawakata, and H. Honjo for fruitful comments and discussions. This research has been partly supported by the Japanese Ministry of Education, Culture, Sports, Science and Technology (MEXT), Grant-in-Aid No. 23654134.

Edited by: A. G. Hunt

Reviewed by: K. Eftaxias and two anonymous referees 


\section{References}

Åström, J. A., Herrmann, H. J., and Timonen, J.: Granular Packings and Fault Zones, Phys. Rev. Lett., 84, 638-641, doi:10.1103/PhysRevLett.84.638, 2000.

Åström, J. A., Herrmann, H. J., and Timonen, J.: Fragmentation dynamics within shear bands-a model for aging tectonic faults?, Eur. Phys. J. E, 4, 273-279, 2001.

Baddari, K. and Frolov, A.: Regularities in discrete hierarchy seismo-acoustic mode in a geophysical field, Ann. Geophys., 53, 31-42, doi:10.4401/ag-4725, 2011.

Bak, P., Christensen, K., Danon, L., and Scanlon, T.: Unified Scaling Law for Earthquakes, Phys. Rev. Lett., 88, 178501, doi:10.1103/PhysRevLett.88.178501, 2002.

Dimiduk, D. M., Woodward, C., LeSar, R., and Uchic, M. D.: ScaleFree Intermittent Flow in Crystal Plasticity, Science, 312, 1188, doi:10.1126/science.1123889, 2006.

Duran, J.: Sands, powders, and grains: An introduction to the physics of granular materials, Springer, New York, 2000.

Eftaxias, K. and Potirakis, S. M.: Current challenges for preseismic electromagnetic emissions: shedding light from micro-scale plastic flow, granular packings, phase transitions and self-affinity notion of fracture process, arXiv:1301.1045, 2013.

Grosse, C. U. and Ohtsu, M. (Eds.): Acoustic Emission Testing, Springer, Berlin, 2008.

Gutenberg, B. and Richter, C. F.: Seismicity of the earth and associated phenomena, Princeton Univ. Press, Princeton, 1949.

Hatano, T. and Kuwano, O.: Origin of the Velocity-Strengthening Nature of Granular Friction, Pure and Appl. Geophys., 170, 3 11, 2013.

Hatano, T., Narteau, C., and Shebalin, P.: Common dependence on stress for the statistics of granular avalanches and earthquakes, arXiv:1110.1777, 2011.

Hidalgo, R. C., Grosse, C. U., Kun, F., Reinhardt, H. W., and Herrmann, H. J.: Evolution of Percolating Force Chains in Compressed Granular Media, Phys. Rev. Lett., 89, 205501, doi:10.1103/PhysRevLett.89.205501, 2002.

Hill, G., Yeung, S., and Koehler, S. A.: Scaling vertical drag forces in granular media, EPL (Europhysics Letters), 72, 137, doi:10.1209/epl/i2005-10203-3, 2005.

Imaeda, H., Kusanagi, H., Kimura, H., and Nakasa, H.: Acoustic emission characteristics durin tensile tests of various metals with several types of crystal structures, The 3rd Acoustic Emission Symposium, Tokyo, 492-512, 1976.

Janssen, H. A.: Versuche über Getreidedtruck in Silozellen, Z. Ver. Dtsh. Ing., 39, 1045-1049, 1895.

Jia, X.: Codalike Multiple Scattering of Elastic Waves in Dense Granular Media, Phys. Rev. Lett., 93, 154303, doi:10.1103/PhysRevLett.93.154303, 2004.

Jia, X., Caroli, C., and Velicky, B.: Ultrasound Propagation in Externally Stressed Granular Media, Phys. Rev. Lett., 82, 1863-1866, doi:10.1103/PhysRevLett.82.1863, 1999.

Kamrin, K. and Koval, G.: Nonlocal Constitutive Relation for Steady Granular Flow, Phys. Rev. Lett., 108, 178301, doi:10.1103/PhysRevLett.108.178301, 2012.

Kapiris, P. G., Balasis, G. T., Kopanas, J. A., Antonopoulos, G. N., Peratzakis, A. S., and Eftaxias, K. A.: Scaling similarities of multiple fracturing of solid materials, Nonlin. Processes Geophys., 11, 137-151, doi:10.5194/npg-11-137-2004, 2004.
Katsuragi, H.: Nonlinear wall pressure of a plunged granular column, Phys. Rev. E, 85, 021301, doi:10.1103/PhysRevE.85.021301, 2012a.

Katsuragi, H.: Material, preparation, and cycle dependence of pressure behavior in a slowly plunged granular column, Chem. Eng. Sci., 76, 165-172, doi:10.1016/j.ces.2012.04.027, 2012b.

Katsuragi, H. and Durian, D. J.: Unified force law for granular impact cratering, Nature Phys., 3, 420-423, doi:10.1038/nphys583, 2007.

Katsuragi, H. and Durian, D. J.: Drag force scaling for penetration into granular media, Phys. Rev. E, 87, 052208, doi:10.1103/PhysRevE.87.052208, 2013.

Kun, F., Lenkey, G. B., Takács, N., and Beke, D. L.: Structure of Magnetic Noise in Dynamic Fracture, Phys. Rev. Lett., 93, 227204, doi:10.1103/PhysRevLett.93.227204, 2004.

Kuwano, O. and Hatano, T.: Flash weakening is limited by granular dynamics, Geophys. Res. Lett., 38, L17305, doi:10.1029/2011GL048530, 2011.

Mehta, A.: Granular physics, Cambridge Univ. Press, Cambridge, 2007.

Mogi, K.: Magnitude-Frequency Relation for Elastic Shocks Accompanying Fractures of Various Materials and Some Related Problems in Earthquakes, Bull. Earthquake Res. Inst., 40, 831853, 1962.

Mogi, K.: Experimental rock Mechanics, Taylor and Francis, London, 2007.

Mori, Y., Obata, Y., Pavelka, J., and Sikula, J.: Ae Kaizer effect and electromagnetic emission in the deformation of rock sample, $\mathrm{J}$ Acoustic Emission, 22, 91-101, 2004.

Mori, Y., Obata, Y., and Sikula, J.: Acoustic and electromagnetic emission from crack created in rock sample under deformation, J. Acoustic Emission, 27, 157-166, 2009.

Olami, Z., Feder, H. J. S., and Christensen, K.: Self-organized criticality in a continuous, nonconservative cellular automaton modeling earthquakes, Phys. Rev. Lett., 68, 1244-1247, doi:10.1103/PhysRevLett.68.1244, 1992.

Owens, E. T. and Daniels, K. E.: Sound propagation and force chains in granular materials, EPL (Europhysics Letters), 94, 54005, doi:10.1209/0295-5075/94/54005, 2011.

Owens, E. T. and Daniels, K. E.: Acoustic measurement of a granular density of modes, Soft Matter, 9, 1214-1219, 2013.

Ramos, O.: Scale Invariant Avalanches: A Critical Confusion, in: Horizons in Earth Science Research. Vol. 3, 157-188, Nova Science Publishers, Inc., 2011.

Richeton, T., Weiss, J., and Louchet, F.: Breakdown of avalanche critical behaviour in polycrystalline plasticity, Nature Mater., 4, 465-469, doi:10.1038/nmat1393, 2005.

Sato, H. and Fehler, M. C.: Seismic Wave Propagation and Scattering in the Heterogeneous Earth, Springer, Berlin, 1997.

Scholz, C. H.: The frequency-magnitude relation of microfracturing in rock and its relation to earthquakes, Bull. Seismol. Soc. Am., 58, 399-415, 1968.

Scholz, C. H.: The mechanics of Earthquakes and Faulting, 2nd Edn., Cambridge Univ. Press, New York, 2002.

Sperl, M.: Experiments on corn pressure in silo cells - translation and comment of Janssen's paper from 1895, Granular Matter, 8, 59-65, doi:10.1007/s10035-005-0224-z, 2006.

Stone, M. B., Barry, R., Bernstein, D. P., Pelc, M. D., Tsui, Y. K., and Schiffer, P.: Local jamming via pene- 
tration of a granular medium, Phys. Rev. E, 70, 041301, doi:10.1103/PhysRevE.70.041301, 2004.

Turcotte, D. L.: Fractals and chaos in geology and geophysics, 2nd edition, Cambridge Univ. Press, New York, 1997.

Yamada, Y., Yamashita, Y., and Yamamoto, Y.: Submarine landslides at subduction margins: Insights from physical models, Tectonophysics, 484, 156-167, 2010.
Yamaguchi, T., Morishita, M., Doi, M., Hori, T., Sakaguchi, H., and Ampuero, J.-P.: Gutenberg-Richter's law in sliding friction of gels, J. Geophys. Res., 116, B12306, doi:10.1029/2011JB008415, 2011.

Zang, A., Wagner, C. F., and Dresen, G.: Acoustic emission, microstructure, and damage model of dry and wet sandstone stressed to failure, J. Geophys. Res., 101, 17507-17521, doi:10.1029/96JB01189, 1996. 